\title{
Trichotillomania: a psychopathological perspective and the psychiatric comorbidity of hair pulling
}

\author{
Sarah Anwar ${ }^{1}$, Mohammad Jafferany ${ }^{\circledR}$
}

\begin{abstract}
Trichotillomania, or hair-pulling disorder, is classified as an obsessive-compulsive spectrum disorder and is seen predominantly in females. This is a non-systematic review article focusing on the psychopathological features of hair pulling. It is speculated that hair pulling may function to provide short-term relief from stress and other unwanted emotional states, thus serving as a method of emotion regulation. The prevalence of trichotillomania ranges from 1 to $3 \%$. The most targeted site is the scalp, and other common areas include pubic hair and facial regions such as the eyebrows, eyelashes, and beard. Individuals suffering from this disorder tend to avoid social environments due to embarrassment regarding their appearance and fears of being judged by peers. Trichotillomania is associated with significant functional impairment and increased risks of comorbid psychiatric disorders such as other body-focused repetitive behaviors, depression, anxiety, and addictive disorders. This article reviews the epidemiology, clinical features, diagnostic criteria, and psychopathology of trichotillomania with an emphasis on psychopathology and psychiatric comorbidity.
\end{abstract}

Keywords: trichotillomania, hair pulling, psychodermatology, psychopathology

Received: 7 August 2018 | Returned for modification: 16 November 2018 | Accepted: 29 October 2018

\section{Introduction}

Trichotillomania, or hair-pulling disorder, is classified as an obsessive-compulsive spectrum disorder. It involves repeated urges to remove one's body hair, resulting in hair loss. Hair-pulling behavior is often preceded by feelings of distress and results in temporarily relief (1). However, the revised diagnostic criteria as set by the fifth edition of the Diagnostic and Statistical Manual of Mental Disorders has removed the feelings of gratification (2). The most common sites reported are the scalp, eyelashes, eyebrows, beard, and pubic hair (3). Hair pulling often involves one or more body areas and may take place for consecutive hours or shorter intervals that appear throughout the day. Pulling generally occurs when alone as opposed to social environments, and many studies have demonstrated that the vast majority of those suffering from trichotillomania report feelings of unattractiveness, humiliation, and low self-confidence (4).

Trichotillomania is often associated with significant distress and functional impairment. Many that suffer from this condition feel embarrassed about their hair loss and tend to avoid social situations due to fear of being judged by their peers (5). Overall, hair pulling may create a cycle in which stress and other undesirable emotions that accompany the hair loss directly correlate with urges to pull the hair and, consequently, a decrease in quality of life.

Recent studies have led to the identification of two distinctive hair-pulling styles, automatic and focused. Automatic pulling occurs with little or no awareness. Many that participate in automatic pulling are unaware of this behavior until they are faced with unwanted consequences such as a new bald spot or handful of hair (6). Conversely, individuals that engage in focused pulling tend to be aware of this activity and may pull to reduce stress or for temporary feelings of pleasure that may accompany the pulling behavior. Most that suffer from trichotillomania have been shown to engage in both automatic and focused pulling (7). A thorough clinical exam and trichoscopy are the main methods for diagnosing trichotillomania.

\section{Epidemiology}

Although few epidemiological studies of trichotillomania exist, recent community findings have estimated the life prevalence of this condition to be between 1 and $3 \%$, with a significant female predominance (8). However, disagreements exist regarding the exact sex ratio because some studies suggest that women dominate 9:1 for this disorder whereas others suggest that the sex ratio is actually closer to 4:1 (4). However, the sex distribution in children has been found to be almost identical (9).

Although little information is available on the physiological developments associated with trichotillomania, the presence of a familial component has been identified, with approximately $34.8 \%$ of patients reporting a family history of trichotillomania (10). Multiple findings have demonstrated an increased risk of trichotillomania in first-degree relatives as well as greater risks for accompanied anxiety disorders and other body-focused repetitive behaviors. A recent family study has confirmed that first-degree relatives of patients have increased risks for repeated hair-pulling behavior (11). Moreover, a significant proportion of individuals with trichotillomania have another current psychiatric diagnosis or another lifetime (present and/or past) psychiatric diagnosis. Specifically, trichotillomania showed substantial overlap with depressive, anxiety, addictive, and other body-focused repetitive behavior disorders.

\section{Clinical features}

The average age of onset for trichotillomania is 12, with this disorder most commonly first seen between ages 10 to 13 (12). Hair pulling generally takes place in one or more areas, with the most common 
site being the scalp, and other popular regions being pubic hair and facial hair such as the eyebrows, eyelashes, and beard (6). Recent studies suggest that children develop more focused pulling and pull from an increasing number of regions as they become older (9).

Trichotillomania is most frequently seen in females, who also face other body-focused repetitive behavior disorders such as nail biting, cheek biting, and skin picking (3). In addition, patients tend to experience comorbid psychiatric conditions such as depressive, anxiety, and addictive disorders at a significant rate (9).

Post-pulling behavior varies among individuals. Whereas some discard the hair after it has been removed, others are known to engage in various activities with the removed hair, ranging from examining, playing with, biting, and even swallowing it. Recent research has demonstrated that over $20 \%$ of trichotillomania patients ingest their hair, a practice that can lead to the creation of masses of hair, or trichobezoars, causing undesirable medical issues (13).

Individuals with trichotillomania often experience feelings of stress and embarrassment due to the resulting hair loss. Many avoid social situations because they are very conscious of unwanted characteristics of hair pulling such as bald spots, and they fear judgement from others (7). Hair pulling generally occurs when alone or performing a sedentary activity, and it can form a vicious cycle in which the negative emotions associated with post-pulling encourage continued pulling in hopes of temporary relief (1). Those suffering from this condition face significant risks of anxiety and depression, and almost one-third report a low or very low quality of life (5).

\section{Diagnostic criteria}

According to the DSM-5, the current diagnostic criteria for trichotillomania are as follows: i) recurrent pulling out of one's hair, resulting in hair loss; ii) repeated attempts to decrease or stop hair pulling; iii) the hair pulling causes clinically significant distress or impairment in social, occupational, or other important areas of functioning; iv) the hair pulling or hair loss is not attributable to another medical condition (e.g., a dermatological condition); and $v$ ) the hair pulling is not better explained by the symptoms of another mental disorder (e.g., attempts to improve a perceived defect or flaw in appearance in body dysmorphic disorder) (2). Trichotillomania appears in the ICD-10, in Chapter 5 on mental and behavioral disorders, and it is coded as F63.3.

Few individuals with trichotillomania obtain professional help. Many are unaware that hair pulling is not an uncommon psychiatric condition, feel embarrassed about their appearance, or worry that an effective treatment does not exist. However, without treatment, only about $14 \%$ of adults experience any decrease in symptoms (3).

A diagnosis of trichotillomania is typically made from a psychiatric examination when hair-pulling behavior is suspected or a patient confesses to removing his or her own hair. Any comorbid conditions are also considered, and treatment options are evaluated. However, additional medical testing becomes necessary if a patient admits to ingesting his or her own hair because this activity can lead to the formation of trichobezoars and further medical concerns (7).

Trichoscopy, an examination of hair and scalp regions using a dermatoscope, is a common technique used to identify hair-pulling behavior. Trichotillomania patients often present asymmetri- cal regions of alopecia in addition to fractured, coiled, and short vellus hairs. Sparse yellow dots that sometimes contain remnants of dead hair follicles presenting as black dots also point to a diagnosis of trichotillomania (14).

\section{Psychopathology}

It is speculated that hair pulling may serve as a means to release tension that is generated by various emotional states (1). Pulling may provide temporary relief from negative emotions such as shame, sadness, frustration, anger, anxiety, and boredom. Multiple studies monitoring emotion regulation in individuals with trichotillomania have demonstrated that these individuals struggle to regulate various emotional states when compared to controls (15). Thus, the development of trichotillomania may be a potential behavioral response in order to cope with unwanted negative emotions. Many findings have revealed that decreases in feelings of boredom, stress, and frustration have been seen across the pulling cycle. In addition, temporary increases in pleasure and relief have been found. However, patients have reported increased feelings of shame, sadness, and frustration shortly after hair pulling was finished (16). These data support the idea that pulling may function to reduce unwanted emotions and is thus reinforced, although any feelings of relief are temporary and often result in the previous unwanted emotions, thus creating a vicious cycle.

Trichotillomania patients are known to suffer from comorbid mental disorders at a much greater rate than the general population (6). A recent study on 85 participants revealed that $38.8 \%$ (33 patients) had at least one other current psychiatric disorder and $78.8 \%$ (67 patients) had at least one other lifetime disorder. Depression, anxiety, mood, and addictive disorders were most frequently presented in this study (17). However, while research concerning the numerical presence of comorbid conditions has been consistent, there have been discrepancies regarding the frequency of specific conditions. A clinical sample demonstrated that $28.6 \%$ of patients suffered from major depression and $10.7 \%$ experienced obsessive-compulsive disorder (OCD), although previous studies have documented higher rates of both comorbid conditions (7). In addition, it has been reported that approximately $70 \%$ of individuals face another body-focused repetitive disorder such as skin picking or nail biting (18).

Differences in the frequency of hair-pulling urges and pulling styles as children age indicate that trichotillomania may follow a developmental progression. Children are known to face more urges to pull and spend more time pulling as they get older (9). However, this positive correlation between age and pulling solely involves focused pulling. It is speculated that children become more aware of pulling urges as they become older. However, it is uncertain whether children face increased urges as they age or are more likely to recognize these urges. Multiple studies have considered a relationship between ages of biological changes in both children and adults with increases in focused pulling and functional impairment (8). These findings indicate that the reason behind increased pulling urges as children age is the onset of puberty. Similarly, adult women have been seen to experience more significant pulling urges during the years that directly precede menopause, further supporting the claim that biological changes may be associated with focused pulling (19).

Recent findings reveal that personality traits may serve as a predictive factor for a trichotillomania diagnosis as well as pulling intensity and styles. A strong correlation was demonstrated 
between neuroticism and a trichotillomania diagnosis with each one-point increase in neuroticism scores serving as a 10\% higher chance of a diagnosis. Higher neuroticism was also linked to stronger pulling intensity and increased focused pulling (20). No relation between the other NEO traits and a diagnosis has been found, and none of the traits predicted automatic pulling. However, higher openness and lower agreeableness are also known to be related to greater pulling intensity, and lower openness is associated with more focused pulling (21).

The relationship between childhood trauma and violence has been a subject of interest in recent years, and one study has demonstrated that $91 \%$ of patients experienced trauma or violence at some point throughout their lives. The vast majority of these episodes involved familial abuse ranging from verbal abuse to physical and sexual assault. Furthermore, $86 \%$ of these individuals believed that their traumatic experiences were related to their first memories of pulling because the hair-pulling behavior occurred within a year of the violent episodes in each case (22). These patients faced significant childhood trauma, suggesting that distressing experiences may play a role in the development of trichotillomania. Other studies have shown that approximately 76 to $86 \%$ of patients have experienced at least one traumatic life event and 19\% have comorbid post-traumatic stress disorder (PTSD), which is an occurrence much greater than that found in the general population. However, a decrease in PTSD symptoms has been observed with a prolonged duration of trichotillomania (10). Thus, trichotillomania may serve as a means to allow patients to cope with disturbing thoughts regarding previous traumatic events.

A high comorbidity of trichotillomania, pathologic skin picking, and OCD has been found, and these behaviors have been labeled as grooming disorders in a recent family study due to their overlap in characteristics of repetitive behaviors. The rates of both trichotillomania patients with comorbid OCD and OCD patients that suffer from trichotillomania are higher than those found in the general population, and it has since been speculated that grooming disorders may occupy a subgroup of OCD (23). Although limited research exists on the relationship between OCD and trichotillomania, it is believed that about 5 to $30 \%$ of trichotillomania patients suffer from OCD, with a recent study stating this value to be $18.9 \%$ (24). Similarly, approximately 5 to $7 \%$ of individuals with OCD are known to experience trichotillomania (25).

Trichotillomania is often associated with depressive, anxiety, and addictive disorders in adults. Recent studies exploring the relationship between depressive and anxiety disorders in children have discovered that almost $50 \%$ of children experience symptoms of depression or anxiety. The findings from one study demonstrate that children that develop trichotillomania later in childhood tend to exhibit an increased number and intensity of depressive symptoms. In this sample, approximately $50 \%$ of teenagers faced significant feelings of depression compared to only $17 \%$ of younger children exhibiting depressive symptoms (9). It has been hypothesized that those with a later onset of trichotillomania, specifically teenagers, are more embarrassed about hair loss and receiving judgement from peers, whereas those with an earlier onset may have developed techniques to avoid feelings of shame or may be better able to conceal their trichotillomania from others (8). In addition, a positive correlation has been shown between worse trichotillomania symptoms and more intense depressive symptoms (17).

Recent research suggests that sex may also be a critical clinical aspect of identifying and treating trichotillomania. Although the demographics of men and women with this disorder tend to be remarkably consistent, there is little agreement on differences in age of onset, functional impairment, and rates of comorbidity (19). Although some findings indicate that men have a later age of onset, report higher levels of functional impairment, and have similar comorbidity as women, more recent findings suggest that age of onset, number of pulling sites, and time spent pulling does not vary between the sexes, but females report greater functional impairment and experience greater psychiatric comorbidity (8).

\section{Treatment}

Although no FDA-approved medication for trichotillomania currently exists, habit reversal therapy in combination with pharmacological treatment has demonstrated promise. Habit reversal therapy sessions generally occur weekly and involve self-monitoring, awareness and prevention training, and stimulus control techniques. Although this form of treatment was often accompanied by anti-depressants and anti-psychotics in the past, $\mathrm{N}$ acetylcysteine has since gained recognition for its effectiveness in treatment for adults with trichotillomania (26). Swedo et al. reported that clomipramine appears to be effective in the short-term treatment of trichotillomania (27). Trichotillomania has also been successfully treated with risperidone and naltrexone in a geriatric case report (28). Other potential non-pharmacological treatments include psychoanalysis, cognitive-behavioral therapy, acceptance and commitment therapy, and dialectical behavioral therapy (29).

\section{Conclusion}

Trichotillomania is a psychodermatological condition associated with hair-pulling behavior that is seen predominantly in women. Individuals with this disorder tend to face significant functional impairment and are known to suffer from comorbid disorders such as other body-focused repetitive behaviors, depression, anxiety, and addictive disorders at significant rates.

Hair pulling may offer temporary relief from undesirable feelings, thus serving as a method of emotion regulation. Many patients were victims of childhood trauma and violence, and it is believed that the development of trichotillomania may provide a technique to manage intrusive thoughts pertaining to traumatic events. Although habit reversal therapy is the mainstay of treatment, newer pharmacological treatments such as $\mathrm{N}$-acetylcysteine are being tried with variable results. Selective serotonin reuptake inhibitors, mood stabilizers, and antipsychotic medications have also been successful in some case reports and open label trials. Collaboration between psychiatrists and dermatologists is crucial in the diagnosis and treatment of trichotillomania in patients. 


\section{References}

1. Roberts S, O’Connor K, Bélanger C. Emotion regulation and other psychological models for body-focused repetitive behaviors. Clin Psychol Rev. 2013;33:74562.

2. American Psychiatric Association. Diagnostic and statistical manual of mental disorders. 5th ed. Washington, DC: American Psychiatric Association; 2013. Obsessive-compulsive and related disorders; p. 129-33.

3. Grant JE, Chamberlain SR. Trichotillomania. Am J Psychiatry. 2016;173:868-74.

4. Bottesi G, Cerea S, Razzetti E. Investigation of the phenomenological and psychopathological features of trichotillomania in an Italian sample. Front Psychol. 2016;7:256.

5. Odlaug BL, Kim SW, Grant JE. Quality of life and clinical severity in pathological skin picking and trichotillomania. J Anxiety Disord. 2010;24:823-9.

6. Duke DC, Keeley ML, Geffken GR, Storch EA. Trichotillomania: a current review. Clin Psych Rev. 2010;30:181-93.

7. Woods DW, Houghton DC. Diagnosis, evaluation, and management of trichotillomania. Psychiatr Clin North Am. 2014;37:301-17.

8. Panza KE, Pittenger C, Bloch MH. Age and gender correlates of pulling in pediatric trichotillomania. J Am Acad Child Adolesc Psychiatry. 2013:52:241-9.

9. Lewin AB, Piacentini J, Flessner CA, Woods DW, Franklin ME, Keuthen NJ, et al. Depression, anxiety, and functional impairment in children with trichotillomania. Depress Anxiety. 2009;26:521-7.

10. Özten E, Sayar GH, Eryilmaz G, Kagan G, Isik S, Karamustafalioglu O. The relationship of psychological trauma with trichotillomania and skin picking. Neuropsychiatr Dis Treat. 2015;11:1203-10.

11. Keuthen NJ, Altenburger EM, Pauls D. A family study of trichotillomania and chronic hair pulling. Am J Med Genet B Neuropsychiatr Genet. 2014;165:167-74.

12. Szepietowski JC, Salomon J, Pacan P, Hrehorów E, Zalewska A. Frequency and treatment of trichotillomania in Poland. Acta Derm Venereol. 2009;89:267-70.

13. Grant JE, Odlaug BL. Clinical characteristics of trichotillomania with trichophagia. Compr Psychiatry. 2008;49:579-84

14. Abraham LS, Torres FN, Azulay-Abulafia L. Dermoscopic clues to distinguish trichotillomania from patchy alopecia areata. An Bras Dermotol. 2010;85:723-6.

15. Shusterman A, Feld L, Baer L, Keuthen N. Affective regulation in trichotillomania: evidence from a large-scale internet survey. Behav Res Ther. 2009;47:637-44.

16. Bottesi G, Cerea S, Ouimet AJ, Sica C, Ghisi M. Affective correlates of trichotillomania across the pulling cycle: findings from an Italian sample of self-identified hair pullers. Psychiatry Res. 2016;246:606-11.

17. Houghton DC, Maas J, Twohig MP, Saunders SM, Compton SN, Neal-Barnett AM, et al. Comorbidity and quality of life in adults with hair pulling disorder. Psychiatry Res. 2016;239:12-9.
18. Stein DJ, Grant JE, Franklin ME, Keuthen N, Lochner C, Singer HS, et al. Trichotillomania (hair pulling disorder), skin picking disorder, and stereotypic movement disorder: toward DSM-V. Depress Anxiety. 2010;27:611-26.

19. Flessner CA, Woods DW, Franklin ME, Keuthen NJ, Piacentini J. Cross-sectional study of women with trichotillomania: a preliminary examination of pulling styles, severity, phenomenology, and functional impact. Child Psychiatr Hum Dev. 2009;40:153-67.

20. Keuthen NJ, Tung ES, Altenburger EM, Blais MA, Pauls DL, Flessner CA. Trichotillomania and personality traits from the five factor model. Rev Bras Psiquiatr. 2015;37:317-24.

21. Keuthen NJ, Tung ES, Tung MG, Curley EE, Flessner CA. NEO-FFI personality clusters in trichotillomania. Psychiatry Res. 2016;239:196-203.

22. Boughn S, Holdom JJ. The relationship of violence and trichotillomania. J Nurs Scholarsh. 2003;35:165-70.

23. Lovato L, Ferrao YA, Stein DJ. Skin picking and trichotillomania in adults with obsessive-compulsive disorder. Compr Psychiatry. 2012;53:562-8.

24. Greenberg E, Grant JE, Curley EE, Lochner C, Woods DW, Tung ES, et al. Predic tors of comorbid eating disorders and association with other obsessive-compulsive spectrum disorders in trichotillomania. Compr Psychiatry. 2017;78:1-8.

25. Torresan RC, Ramos-Cerqueira AT, Shavitt RG, do Rosário MC, de Mathis MA, Miguel EC, et al. Symptom dimensions, clinical course and comorbidity in men and women with obsessive compulsive disorder. Psychiatry Res. 2013;209:18695 .

26. Castillo D, Enos C, Franca K, et al. Pharmacotherapy. In: Franca K, Jafferany M, editors. Trichotillomania (hair pulling disorder): clinical characteristics, psychological interventions and emotional effects. New York: Nova Science Publishers; 2015. p. 55-74.

27. Swedo SE, Leonard HL, Rapoport JL, Lenane MC, Goldberger EL, Cheslow DL. A double-blind comparison of clomipramine and desipramine in the treatment of trichotillomania (hair pulling). N Eng J Med. 1989;321:497-501.

28. Oravecz $R$, Stuhec $M$. Trichotillomania successfully treated with risperidone and naltrexone: a geriatric case report. J Am Med Dir Assoc. 2014;15:301-2.

29. Shenefelt, PD. Non-pharmacological treatments for trichotillomania. In: Franca $\mathrm{K}$, Jafferany M, editors. Trichotillomania (hair pulling disorder): clinical characteristics, psychological interventions and emotional effects. New York: Nova Science Publishers; 2015. p. 75-84. 